\title{
Inevitability of the Poisson Bracket Structure of the Relativistic Constraints
}

\author{
Jan Głowacki ${ }^{1,2}$ (D)
}

Received: 8 January 2021 / Accepted: 15 October 2021 / Published online: 5 November 2021

(c) The Author(s) 2021

\begin{abstract}
The purpose of this paper is to shed some fresh light on the long-standing conceptual question of the origin of the well-known Poisson bracket structure of the constraints that govern the canonical dynamics of generally relativistic field theories, i.e. geometrodynamics. This structure has long been known to be the same for a wide class of fields that inhabit the space-time, namely those with non-differential coupling to gravity. It has also been noticed that an identical bracket structure can be derived independently of any dynamical theory, by purely geometrical considerations in Lorentzian geometry. Here we attempt to provide the missing link between the dynamics and geometry, which we understand to be the reason for this structure to be of the specific kind. We achieve this by a careful analysis of the geometrodynamical approach, which allows us to derive the structure in question and understand it as a consistency requirement for any such theory. In order to stay close to the classical literature on the subject we stick to the metric formulation of general relativity, but the reasoning should carry over to any other formulation as long as the non-metricity tensor vanishes. The discussion section is devoted to derive some interesting consequences of the presented result in the context of reconstructing the Arnowitt-Deser-Misner (ADM) framework, thus providing a precise sense to the inevitability of the Einstein's theory under minimal assumptions.
\end{abstract}

Keywords Relativistic constraints · Poisson bracket · Geometrodynamics

Jan Głowacki

glowacki@cft.edu.pl

1 Center for Theoretical Physics, Polish Academy of Sciences, Al. Lotników 32/46, 02-668 Warsaw, Poland

2 Basic Research Community for Physics, Mariannenstraße 89, 04-315 Leipzig, Germany 


\section{Introduction}

In this paper we are concerned with the Hamiltonian perspective and hence we consider only globally-hyperbolic space-times. Moreover, since we assume the ADM Hamiltonian to split for the gravity and the matter parts, we assume the matter-gravity coupling to be non-derivative. ${ }^{1}$ Our aim is to show that under these assumptions, i.e. when we assume our theory of gravity to be of the form of geometrodynamics as presented in Sect. 3 (which is true for any matter field with non-derivative coupling to gravity), and restrict our attention to fully deterministic scenarios (global hyperbolicity), the Poisson brackets between the relevant super-Hamiltonian and super-momentum are actually fixed as a consequence of the compatibility condition (Postulate, i.e. the Theorem 1 below) which, as we argue, does nothing more than assuring the covariant and canonical pictures to carry the same physical information.

One of the consequences of our considerations, which we discuss in the last part of the paper, is strengthening the reconstruction result of [1], where the vacuum gravitational constraints are constructed under the assumption (among other) that the Poisson bracket structure of the super-Hamiltonian and super-momentum is of the desired form. Using our result, this assumption can be replaced by our compatibility postulate, which we find almost unavoidable.

The particular Poisson bracket structure of the constraints governing the evolution of gravity + matter ADM systems is interesting for at least three related reasons:

(i) it is independent of the types of fields considered (as long as the coupling to gravity is non-derivative),

(ii) it seems to have a purely geometrical origin,

(iii) it almost suffices to deduce the particular form of the gravitational constraints.

The first point is an intriguing observation made by considering gravity + matter systems in the ADM framework under the assumption that the coupling term does not include derivatives of the metric [1]. The second is an indication that follows from successful attempts to derive an identical structure from purely geometrical considerations, namely deformations of hypersurfaces embedded in Lorentzian manifolds (see [2-4]).

In the most recent approach of [3] a groupoid is proposed whose algebroid bracket structure of constant sections is equivalent to the Poisson bracket structure of the constraints. However, as the authors put it ([3], p. 3):

\footnotetext{
1 We follow here [1], see Eq. 2.10 on p. 97. Non-derivative couplings are standard and include in particular the Einstein-Yang-Mills (and hence also Einstein-Maxwell) theories, since the Yang-Mills functional that is responsible for coupling does not contain derivatives of the metric. Let us also note here, that even though we are concerned only with the super-Hamiltonian and super-momentum constraints, it does not mean that those are the only constraints that the fields are subject to-there may well be more constraints that could be found e.g. by returning to the Lagrangian picture and differentiating the matter Lagrangian with respect to the matter velocities.
} 
It remains to be seen what the relevant structure is which connects our groupoid with the constraints themselves.

This is the very question we would like to propose an answer to. ${ }^{2}$ The third reason named above is the claim of [1], where the constraints of pure gravity are recovered under the assumption that their Poisson bracket closes in this particular way. We will reflect on this in the light of our result in the discussion.

\section{Canonical General Relativity}

\subsection{The Constraints}

The constraints of general relativity arise when the Legendre transform is performed on the Lagrangian of the projected Einstein-Hilbert action, possibly coupled (in a non-derivative way) to some matter fields [6]:

$$
\begin{aligned}
S & =\int_{M} \sqrt{-g}\left\{{ }^{4} \mathrm{R}(g)+\mathscr{L}_{\text {mat }}\right\} \\
& =\int_{\mathbb{R}} d \tau \int_{\Sigma_{\tau}} \operatorname{vol}_{\gamma_{\tau}}\left\{{ }^{3} \mathrm{R}\left(\gamma_{\tau}\right)+\operatorname{tr}\left(k_{\tau}^{2}\right)-\operatorname{tr}\left(k_{\tau}\right)^{2}+\left(\mathrm{L}_{\text {mat }}\right)_{\tau}\right\},
\end{aligned}
$$

where:

- the slicing of the globally hyperbolic space-time manifold $M$ is chosen so that $M \cong \Sigma \times \mathbb{R}$ and each slice $\Sigma_{\tau}$ is a Cauchy surface,

- $\gamma_{\tau}$ is the first fundamental form on $\Sigma_{\tau}$, i.e. Riemannian 3-metric inherited from $M$, and

- $k_{\tau}$ is the second fundamental form of $\Sigma_{\tau}$, i.e. its extrinsic curvature, given by $k_{\tau}=-\frac{1}{2} \mathscr{L}_{\hat{n}_{\tau}} \gamma$.

The choice of the slicing $M \cong \Sigma \times \mathbb{R}$ is equivalent to picking an initial value Cauchy surface $\Sigma_{0} \subset M$ and a time-like evolution vector field $E \in \mathscr{X}(M)$ on the space-time $M$. Indeed, since $\Sigma_{0}$ is Cauchy and $M$ is globally hyperbolic, any endless (i.e. with globally defined flow) time-like curve on $M$ will cross $\Sigma_{0}$ exactly once, and hence the flow $\alpha_{E}^{\tau}$ of $E$ defines a foliation of $M$ via $\Sigma_{\tau}:=\alpha_{E}^{\tau}\left(\Sigma_{0}\right)$. Conversely, making the topological slicing $M \cong_{t o p} \Sigma \times \mathbb{R}$ an isometry requires choosing the way in which the neighbouring slices are to be glued together into a Lorentzian manifold, which can be achieved by choosing a real positive function $L_{\tau} \in \mathscr{F}_{+}\left(\Sigma_{\tau}\right)$, called the lapse, and a vector field $\vec{N}_{\tau} \in \mathscr{X}\left(\Sigma_{\tau}\right)$, called the shift, on each slice, which in turn defines the evolution vector field on the whole $M$ via:

\footnotetext{
2 The authors are pursuing their own direction aiming at understanding this connection by developing the framework of Hamiltonian Lie algebroids [5]. This is interesting on its own but very different from the relatively simple approach presented here.
} 


$$
\left.E\right|_{\Sigma_{\tau}}=\vec{N}_{\tau}+L_{\tau} \hat{n}_{\tau} .
$$

Since all the Cauchy slices are diffeomorphic, we could as well consider parametrized families of tensors, functions and vector fields defined simply on $\Sigma$. This is the point of the ADM approach: it allows to consider the solution of the Einstein equation, i.e. the space-time with all the relevant tensor fields, to be generated by the initial data given solely on $\Sigma$, together with the choice of the lapse and the shift.

Notice here that what we call the 'speed' of $\gamma$, i.e. the second fundamental form $k$, is given by the Lie derivative with respect to the direction normal to the slice, which plays the role of the local time direction (justifying the terminology). Since any oriented space-like hypersurface admits a neighbourhood, called a (local) Gaussian neighbourhood, where the coordinates arise via drawing geodesics that start on the surface and aim to the positively oriented direction with the initial speed given by the normal vectors at each point, this quantity is always unambiguously defined. The described construction can be seen as the canonical way of 'smearing out' the hypersurface and defining the (local) time direction, referred to as the (local) Gaussian time, with respect to which the 'speeds' of all the fields at a given slice are naturally defined. The resulting coordinates are called (local) Gaussian normal coordinates. The projected 3-dimensional matter Lagrangian $L_{\text {mat }}$ is then also a functional of the fields on $\Sigma$, changing with the $\tau$ parameter, whose 'speeds' are given through the Lie derivative in the normal direction at each slice, which are therefore also $\tau$ -dependent.

Neglecting the boundary terms and under the assumption that the gravity-matter coupling is non-derivative, the Legendre transformation gives rise to the ADM Hamiltonian of the form [1]:

$$
\begin{aligned}
H_{E}(\tau) & =\left.\int_{\Sigma_{\tau}} \operatorname{vol}_{\gamma_{\tau}} g\left(E, V_{H}\right)\right|_{\Sigma_{\tau}} \\
& =\int_{\Sigma_{\tau}} \operatorname{vol}_{\gamma_{\tau}}\left(-L_{\tau} \mathscr{H}+\gamma_{\tau}\left(\overrightarrow{\mathscr{H}}, \vec{N}_{\tau}\right)\right)=H_{\left(\vec{N}_{\tau}, L_{\tau}\right)},
\end{aligned}
$$

where we have introduced the notation $\left.V_{H}\right|_{\Sigma_{\tau}}:=\overrightarrow{\mathscr{H}}+\mathscr{H} \hat{n}_{\tau}$ with $\mathscr{H}$ being the Hamiltonian constraint and $\overrightarrow{\mathscr{H}}$ the momentum constraint, both depending solely on the fields and their momenta. The ADM Hamiltonian is of the above form for any matter field with non-derivative coupling [1], and this is where we need to use this assumption. The equality (3) holds since on each slice we have $\left.g\right|_{\Sigma_{\tau}}=-d t^{2}+\gamma_{\tau}$ (where $\gamma$ is thought of as extended by zero to a 4-dimensional object and $t$ denotes the local Gaussian time). Let us stress here, that the above decomposition, as the $\tau$-subscript in our notation suggests, can only be achieved on each slice separately-we by no means assume such coordinates to exist in outside an infinitesimal neighbourhood of any of the slices. The minus sign by $L_{\tau}$ in (3) is non-standard, but since $\mathscr{H}=0$ it makes no harm and makes the equality hold, which will prove useful in what follows. We will consistently use the letters $t$ to denote the Gaussian time and $\tau$ for the slicing parameter, i.e. the flow parameter of the evolution vector field. For pure gravity these constraints read [3]: 


$$
\begin{gathered}
\overrightarrow{\mathscr{H}}\left(\gamma_{\tau}, \pi_{\tau}\right)=-2 \operatorname{div}\left(\pi_{\tau}\right), \\
\mathscr{H}\left(\gamma_{\tau}, \pi_{\tau}\right)=-{ }^{3} \mathrm{R}\left(\gamma_{\tau}\right)+\operatorname{tr}\left(\pi_{\tau}^{2}\right)-\frac{1}{2} \operatorname{tr}\left(\pi_{\tau}\right)^{2},
\end{gathered}
$$

where $\pi$ is the variable conjugate to $\gamma$. Defining $H_{\vec{N}_{\tau}}:=H_{\left(\vec{N}_{\tau}, 0\right)}, H_{L_{\tau}}:=H_{\left(0, L_{\tau}\right)}$, the Poisson bracket structure in question can be captured as follows [3]:

$$
\begin{gathered}
\left\{H_{\vec{N}_{\tau}}, H_{\vec{N}_{\tau}^{\prime}}\right\}=H_{\left[\vec{N}_{\tau}, \vec{N}_{\tau}^{\prime}\right]}, \\
\left\{H_{\vec{N}_{\tau}}, H_{L_{\tau}}\right\}=H_{\vec{N}_{\tau}\left(L_{\tau}\right)}, \\
\left\{H_{L_{\tau}}, H_{L_{\tau}^{\prime}}\right\}=H_{L_{\tau} \operatorname{grad}_{\gamma_{\tau}} L_{\tau}^{\prime}-L_{\tau}^{\prime} \operatorname{grad}_{\gamma_{\tau}} L_{\tau}} .
\end{gathered}
$$

Including matter fields results in adding matter terms, depending on the fields and their momenta coupled to the gravitational data, to the constraints. For the matter fields coupled to gravity in a non-derivative way, this does not affect the Poisson bracket structure of the above constraints [1]. The main goal of this paper is to give a conceptual reason for this structure to be inevitable and independent of the particular matter content, thus addressing the properties (i) and (ii) named at the beginning.

\subsection{Geometrodynamics}

The geometrodynamical framework, which is of the main interest here, is most naturally seen as a generalization of the non-relativistic Hamiltonian field theory, which we are now going to briefly review. It is specified by the following data:

- A phase space $\Gamma$, which is an (infinite-dimensional) Poisson manifold consisting of all possible configurations of the fields and their momenta:

$$
\Gamma \ni\left(\varphi_{1}, \ldots, \varphi_{n}, p_{1}, \ldots, p_{n}\right), \quad \varphi_{i}: \Sigma \rightarrow V_{\varphi_{i}}, \quad p_{i}: \Sigma \rightarrow V_{p_{i}},
$$

where the $V \mathrm{~s}$ are the vector spaces where the fields take theirs values, and $\Sigma$ is the (3-dimensional) manifold on which the fields are defined, ${ }^{3}$

- We are concerned with the time evolution of functionals on $\Gamma$ (implicitly dependent on the external time, denoted here by ' $s$ ') of the following form:

$$
A: \Gamma \rightarrow \mathbb{R}, \quad A\left[\varphi_{i}, p_{i}\right](s)=\int_{\Sigma} d^{3} x \mathscr{A}\left(\varphi_{i}(s), p_{i}(s)\right),
$$

- A Hamiltonian $H(s)=\int_{\Sigma} d^{3} x \mathscr{H}\left(\varphi_{i}(s), p_{i}(s)\right)$ generating the dynamics via:

\footnotetext{
${ }^{3}$ In particular, $\Gamma$ depends on the boundary conditions that we impose, but we do not enter the details here and simply assume that all these choices have been made and we have a well-defined field theory.
} 


$$
\frac{d A(s)}{d s}=\{A, H\}=\frac{\delta A}{\delta \varphi_{i}} \frac{\delta H}{\delta p_{i}}-\frac{\delta A}{\delta p_{i}} \frac{\delta H}{\delta \varphi_{i}},
$$

where $\delta$ denotes the functional derivative and the summation convention applies. Such a setup can be generalized to the ' $3+1$ ' diffeomorphism invariant realm, where the 3-dimensional metric on $\Sigma$ is treated as a dynamical variable and the evolution is given with respect to the evolution vector field [1]:

- The phase space is equal $\tilde{\Gamma}=\Gamma \times T^{*} \operatorname{Riem}(\Sigma)=\Gamma \times \operatorname{Riem}(\Sigma) \times S^{2}(\Sigma)$, which is extended by the 3-dimensional metric fields $\gamma$ and their conjugate momenta (symmetric two-forms) $\pi$, so that:

$$
\tilde{\Gamma} \ni\left(\gamma, \varphi_{1}, \ldots, \varphi_{n}, \pi, p_{1}, \ldots, p_{n}\right),
$$

- We are concerned with the evolution of functionals on the (new) phase space, dependent on the slicing parameter:

$$
A\left[\gamma, \pi, \varphi_{i}, p_{i}\right](\tau)=\left(\alpha_{E}^{\tau}\right)^{*} A\left[\gamma_{0}, \pi_{0},\left(\varphi_{i}\right)_{0},\left(p_{i}\right)_{0}\right]=\int_{\Sigma_{\tau}} \operatorname{vol}_{\gamma_{\tau}} \mathscr{A}\left(\gamma_{\tau}, \pi_{\tau},\left(\varphi_{i}\right)_{\tau},\left(p_{i}\right)_{\tau}\right),
$$

- The Hamiltonian depends on the evolution vector field $E(x, \tau)=\left(L_{\tau}(x), \vec{N}_{\tau}(x)\right)$ on $\Sigma \times \mathbb{R}$ in the following way (see Eq. (3)):

$$
\begin{aligned}
H_{E}(\tau)= & \int_{\Sigma_{\tau}} \operatorname{vol}_{\gamma_{\tau}} g\left(E, V_{H}\right)=\int_{\Sigma_{\tau}} \operatorname{vol}_{\gamma_{\tau}} \quad\left(-L_{\tau} \mathscr{H}\right. \\
& \left.+\gamma_{\tau}\left(\vec{H}, \vec{N}_{\tau}\right)\right)=H_{\left(\vec{N}_{\tau}, L_{\tau}\right)}
\end{aligned}
$$

where $\mathscr{H}=\mathscr{H}\left(\gamma, \pi, \varphi_{i}, p_{i}\right), \overrightarrow{\mathscr{H}}=\overrightarrow{\mathscr{H}}\left(\gamma, \pi, \varphi_{i}, p_{i}\right)$ and the evolution equation reads:

$$
\frac{d A(\tau)}{d \tau}=\left.\frac{d}{d \tau}\left(\alpha_{E}^{\tau}\right) * A\right|_{\Sigma_{\tau}}=\left.\mathscr{L}_{E} A\right|_{\Sigma_{\tau}}=\left\{H_{\left(\vec{N}_{\tau}, L_{\tau}\right)}, A\right\},
$$

with the Poisson bracket defined with respect to all field variables (including the $(\gamma, \pi)$ pair).

When no matter fields are present, setting $\mathscr{H}$ and $\overrightarrow{\mathscr{H}}$ accordingly to (4) and (5) gives ADM gravity. For examples of gravity + matter systems with non-differential coupling captured in this form see [1]. It is not known if this particular form of geometrodynamics can capture more general gravity + matter systems. Considering the Minkowski space-time $\mathbb{M}^{4}$ with $E=\frac{\partial}{\partial s}$ gives $(\gamma, \pi)=(\operatorname{diag}(1,1,1), 0)$, $(X, \phi)=(0,1)$, thus allowing to recover the non-relativistic setup (watch the sign).

\subsection{Plan of the Remaining of the Paper}

In the next section we present the main observation concerning the evolution of the initial data with respect to the evolution vector field. A simple unambiguity requirement, namely the Postulate (Theorem 1) below, which takes its precise form in Eq. 
(11), will place a condition on the local behaviour of the evolution vector fields that can consistently be considered. Using the terminology of [3], we argue that the only evolution vector fields that should be considered are $g$-Gaussian with respect to any slice of the foliation. ${ }^{4}$

In the fourth section we derive the Poisson bracket structure of the constraints as a consequence of the mentioned observation in this context. More precisely, by applying the geometrodynamical evolution equation to the Hamiltonian defined with respect to the evolution vector field that is different from the one fixing the dynamics, we prove that the Poisson bracket in question is reflected by the commutator of the relevant evolution vector fields. We identify the generators of the deformations with the lapse and shift of admissible evolution vector fields, which we argue to be $g$-Gaussian on each slice, thus providing the link between the Teitelboim construction of [2], the algebroid approach of [3] and the geometrodynamical constraints. Further, following [3], we calculate this commutator and show that it yields the correct structure.

The discussion is devoted to the reflection on the consequences of the advocated point of view in the light of the reconstruction of geometrodynamics proposed in [1]. We conclude that our result allows to justify the assumption of the particular Poisson bracket structure of the constraints to the point that it should, in our view, no longer be seen as an additional assumption on the top of the geometrodynamical framework, thus considerably strengthening the reconstruction result.

\section{Our Postulate}

In order to transform the fully covariant theory of General Relativity into a dynamical theory we break the 4-dimensional diffeomorphism invariance by assuming global hyperbolicity and fixing the slicing of the space-time with Cauchy surfaces. The initial data are then to be given on such a surface $\Sigma_{1}$, while the evolution equations should predict their values at each point of the space-time, i.e. on any other slice of the foliation. As described in the Sect. 2 above, the 'gravity + matter' action can be written in the Lagrangian form with respect to such a foliation with the dynamical variables being fields on $\Sigma_{0} \cong \Sigma_{\tau}$ and their Lie derivatives with respect the normal directions.

Here comes our crucial and new observation. On the one hand, we view the speeds of the fields as inhabiting the field theoretic configuration space, e.g. $\operatorname{TRiem}(\Sigma) \cong \operatorname{Riem}(\Sigma) \times S^{2}(\Sigma)$ for the gravitational degrees of freedom ${ }^{5}$. The solution of Hamilton's equations, which now need to depend on the evolution vector field to retain diffeomorphism invariance, should give a curve on the configuration space: $\tau \mapsto\left(\gamma_{\tau}, k_{\tau}\right) \in \operatorname{TRiem}(\Sigma)$. The space $\Sigma$ is here just a 3-dimensional manifold, which

\footnotetext{
4 There is a slight change in terminology that we propose to distinguish between the 'good' evolution vector fields and the $g$-Gaussian extensions proposed in [3], see the next section.

${ }^{5}$ Here $\operatorname{Riem}(\Sigma)$ denotes the space of Riemannian 3-metrics and $S^{2}(\Sigma)$ the space of symmetric twoforms on $\Sigma$.
} 
is not embedded in any space-time, like for non-relativistic field theories. But on the other hand, the 'speeds' of the fields are given through the Lie derivatives in the normal direction when the slices are glued together accordingly to the slicing chosen, i.e. when the particular solution in the form of the fields defined over a full 4-dimensional space-time is constructed. Indeed, this is how they come about when we slice a space-time filled with matter fields which satisfies the Einstein's equation. As we will see, the compatibility of these two perspectives places a non-trivial requirement on the behaviour of the admissible evolution vector fields with respect to the spacetime geometry. It can be stated as follows:

Theorem 1 (Postulate) The canonical and the covariant pictures of the generally relativistic field theory need to carry the same physical information. In particular, the dynamical data should coincide with the sliced space-time data.

This means that for any tensor field $T$ over the space-time manifold $M$, any value of the slicing parameter $\tau$, and its small ${ }^{6}$ change $\Delta \tau$ we should have the same output regardless if we:

1. calculate the Lie derivative with respect to the normal vector field of $\Sigma_{\tau}$, denoted by $\mathscr{L}_{\hat{n}_{\tau}} T$, and then allow the 'time' to flow for a little bit along the flow of the evolution vector field $E$ (so that $\Sigma_{\tau}$ will become $\Sigma_{\tau+\Delta \tau}$ ) and then read out the resulting 3-dimensional 'speed', or

2. take the Lie derivative with respect to $\hat{n}_{\tau+\Delta \tau}$ and read out the data straight from $\Sigma_{\tau+\Delta \tau}$.

In mathematical terms our postulate then asserts exactly that for $T, \tau$ and $\Delta \tau$ as above we should always have:

$$
\left.\left(\left(\alpha_{E}^{\Delta \tau}\right)^{*} \mathscr{L}_{\hat{n}_{\tau}} T\right)\right|_{\Sigma_{\tau+\Delta \tau}}=\left.\left(\mathscr{L}_{\hat{n}_{\tau+\Delta \tau}} T\right)\right|_{\Sigma_{\tau+\Delta \tau}},
$$

where $\alpha_{E}^{\Delta \tau}$ denotes the relevant element of the one-parameter group of diffeomorphisms of $M$ associated with the flow of $E$. The above equality holds since both sides of the equation denote the speed of the projected field $T$ at $\tau+\Delta \tau$ in the canonical picture: the left hand side represents the scenario in which the speed is calculated at $\tau$ and evolved to $\tau+\Delta \tau$, while the right hand side denotes the speed calculated straight at $\tau+\Delta \tau$, which clearly mean the same thing to us and thus should be represented by the same mathematical objects. The simple Lemma below is crucial for our derivation of the constraints algebra:

Lemma 1 If the Eq. (11) above is satisfied for any $T$ and $\tau$ and sufficiently small $\Delta \tau$, the following equality of vector fields holds:

\footnotetext{
${ }^{6}$ We need to be a little careful here with the magnitude of $\Delta \tau$ so that the results of both procedures described below can be compared. See also the coming Lemma 1. In the end we will only need this in the limit $\Delta \tau \searrow 0$.
} 


$$
\left(\alpha_{E}^{\Delta \tau}\right)_{*} \hat{n}_{\tau}=\hat{n}_{\tau+\Delta \tau} .
$$

Remark 1 Let us stress here, that the Eq. (12) above is not satisfied in general, and even less it is tautological, as it may seem to be at first sight. This is simply because the two vector fields involved-namely $\hat{n}_{\tau}$ and $\hat{n}_{\tau+\Delta \tau}$ - are defined by different initial conditions: their flows are the future-oriented, time-like geodesics extending the unit normal vector fields given along different slices, and hence are unrelated in general. It would then be a mistake to assume the equation like the one above to hold without any justification, however plausible it may look. Indeed, the Lemma 1 that we are just going to prove serves exactly this purpose-it derives this property for the evolution vector fields that are allowed by our Postulate (Theorem 1). The equation (12) seems so plausible precisely because we think of the local Gaussian times as representing the local time flow, which indeed we can do, but only if the Postulate (Theorem 1) is granted.

Proof For given $\tau$ take $\Delta \tau$ small enough so that both $\Sigma_{\tau}$ and $\Sigma_{\tau+\Delta \tau}$ lie in the intersection of their Gaussian neighbourhoods so that both sides of (11) are well-defined. Now for $p \in \Sigma_{\tau+\Delta \tau}$ we calculate:

$$
\begin{aligned}
\mathscr{L}_{\hat{n}_{\tau+\Delta \tau}} T(p) & =\left(\alpha_{E}^{\Delta \tau}\right)^{*} \mathscr{L}_{\hat{n}_{\tau}} T(p) \\
& =\mathscr{L}_{\hat{n}_{\tau}} T\left(\alpha_{E}^{\Delta \tau}(p)\right) \\
& =\left.\frac{d}{d s}\right|_{s=0}\left(\alpha_{\hat{n}_{\tau}}^{s}\right)^{*} T\left(\alpha_{E}^{\Delta \tau}(p)\right) \\
& =\left.\frac{d}{d s}\right|_{s=0} T\left(\alpha_{E}^{\Delta \tau} \circ \alpha_{\hat{n}_{\tau}}^{s}(p)\right) \\
& =\left.\frac{d}{d s}\right|_{s=0}\left(\alpha_{E}^{\Delta \tau} \circ \alpha_{\hat{n}_{\tau}}^{s}\right)^{*} T(p)=\mathscr{L}_{\left(\alpha_{E}^{\Delta \tau}\right)_{*} \hat{n}_{\tau}} T(p) .
\end{aligned}
$$

Since $T$ was arbitrary, this finishes the proof.

To sum up our considerations up to this point, we have argued that assuming our Postulate (Theorem 1), we can deduce that the evolution vector fields have to satisfy the condition (12) for any $\tau$ and small enough $\Delta \tau$. As already noted, this apparently simple requirement is in fact quite convoluted and might be confusing. Notice for instance, that the normal directions are defined with respect to the slices but depend also on the space-time metric $g$. The matter of (12) being satisfied is thus a complex issue depending on the background geometry of the space-time. ${ }^{7}$

Consider now an evolution vector field $E$ compatible with our Postulate (Theorem 1), and hence satisfying (12). For any vector field $v$ on the neighbourhood of an arbitrary chosen slice $\Sigma_{\tau}$ and a small enough $\Delta \tau$, we then have:

\footnotetext{
7 The question of precise characterization of the evolution vector fields that satisfy our Postulate (Theorem 1) for a given space-time, and hence provide its admissible slicings, is, to the best of our knowledge, not known and should be addressed elsewhere.
} 


$$
\left(\alpha_{E}^{\Delta \tau}\right)^{*} g\left(\hat{n}_{\tau}, v\right)=g\left(\left(\alpha_{E}^{\Delta \tau}\right)_{*} \hat{n}_{\tau},\left(\alpha_{E}^{\Delta \tau}\right)_{*} v\right)=g\left(\hat{n}_{\tau+\Delta \tau},\left(\alpha_{E}^{\Delta \tau}\right)_{*} v\right) .
$$

This means that the inner product with the normal vector field on a given slice will not change when calculated on a slightly later slice, which in the limit $\Delta \tau \rightarrow 0$ yields (see [3], p. 9):

$$
\left.\left(\mathscr{L}_{E} g\right)\left(\hat{n}_{\tau}, v\right)\right|_{\Sigma_{\tau}}=0 .
$$

A vector field that satisfies (14) will be called $\Sigma_{\tau^{-}}$Gaussian. ${ }^{8}$ Therefore, the evolution vector fields compatible with our Postulate (Theorem 1) are $\Sigma_{\tau}$-Gaussian, for all $\tau$. In order not to make the paper too technical, we will omit the proof of the following fact, which can be found in [3] (pp. 8-9).

Proposition 2 A vector field $E$, defined on a neighbourhood of an oriented embedded space-like hypersurface $\Sigma \subset M$, is $\Sigma_{\tau}$-Gaussian iff it satisfies:

$$
\left.\hat{n}(L)\right|_{\Sigma_{\tau}}=0,\left.\quad[\hat{n}, \vec{N}]\right|_{\Sigma_{\tau}}=\operatorname{grad}_{\gamma_{\tau}} L .
$$

where $E=\vec{N}+L \hat{n}_{\tau}$ and $\operatorname{grad}_{\gamma_{\tau}} L$ denotes the gradient of the function $L$ with respect to $\gamma_{\tau}$, the Riemannian 3-metric of $\Sigma_{\tau}$ inherited from $M$.

We can then conclude that the evolution vector fields allowed by our our Postulate (Theorem 1) will satisfy the Eq. (15) above at each slice. ${ }^{9}$ As we will see, this local condition makes it possible to determine the infinitesimal behaviour of the evolution vector fields knowing their values on the slice alone, which will be crucial for our derivation of the constraints bracket in the next section.

\section{Constraints Bracket Derivation}

Notice first that the Hamiltonian can itself be seen as an functional, and hence we can apply to it the evolution Eq. (10). Then, just like in the non-relativistic case, anti-symmetry of the Poisson bracket assures that $H_{E}$ is 'constant' in the sense that it does not evolve with respect to itself. We can however consider another Hamiltonian, arising from a different choice of the evolution vector field, say $H_{E^{\prime}}$. Its evolution with respect to $H_{E}$ is then given by their Poisson bracket, which hence reads:

\footnotetext{
${ }^{8}$ This is to distinguish from the $g$-Gaussian vector fields that are defined in [3]. Those satisfy the Eq. (14) in the neighbourhood of a fixed slice, not merely on the slice.

9 Unlike in [3], we understand the equation and (15) to hold sharply on the slice, and not in the neighbourhood. The g-Gaussian extension of [3] is not a good evolution vector field, for which the just mentioned equations need to hold on each slice separately. In other words, $\gamma$ should not be considered fixed. Alternatively, we see the $g$-Gaussian extension [3] of a vector field given solely on $\Sigma$ simply as a way to determine its behaviour on the infinitesimal neighbourhood of that slice, given that it is $\Sigma$-Gaussian.
} 


$$
\begin{gathered}
\left\{H_{\left(\vec{N}_{\tau}, L_{\tau}\right)}, H_{\left(\vec{N}_{\tau}^{\prime}, L_{\tau}^{\prime}\right)}\right\}=\left.\mathscr{L}_{E} H_{E^{\prime}}\right|_{\Sigma_{\tau}} \\
=\left.\mathscr{L}_{E} \int_{\Sigma_{\tau}} \operatorname{vol}_{\gamma_{\tau}} g\left(E^{\prime}, V_{H}\right)\right|_{\Sigma_{\tau}} \\
=\int_{\Sigma_{\tau}} \operatorname{vol}_{\gamma_{\tau}} E\left(g\left(E^{\prime}, V_{H}\right)\right) \\
=\int_{\Sigma_{\tau}} \operatorname{vol}_{\gamma_{\tau}}\left(g\left(\nabla_{E} E^{\prime}, V_{H}\right)+g\left(E^{\prime}, \nabla_{E} V_{H}\right)\right) \\
=\int_{\Sigma_{\tau}} \operatorname{vol}_{\gamma_{\tau}} g\left(\left[E, E^{\prime}\right], V_{H}\right)=H_{\left[E, E^{\prime}\right]}(\tau)=H_{\left[\left(\vec{N}_{\tau}, L_{\tau}\right),\left(\vec{N}_{\tau}^{\prime}, L_{\tau}^{\prime}\right)\right]},
\end{gathered}
$$

where we have used: the evolution Eq. (16), the definition of the Hamiltonian (17), diffeomorphism-invariance of the 3-dimensional volume form, together with the fact that all slices are diffeomorphic and the Lie derivative of a function is simply acting on it with the vector field (18), metricity of the connection (19), the fact that the covariant derivative of one vector field with respect to another is given by their commutator, and that $\left[E, V_{H}\right]=0$ (since $V_{H}$ is constant on slice as it depends on the field data, and not on the points on $\Sigma_{\tau}$ and $E$ preserves its tangent-normal decomposition) (20), and finally the definition of the geometrodynamical Hamiltonian again.

The Poisson bracket of the constraints (or actually of the constraints paired with a vector field and a function, which are our Hamiltonians) is then given by the commutator of the evolution vector fields with respect to which they are defined. Thanks to Proposition 2 it can be calculated on each slice of the foliation, with the result depending on the slice chosen. Indeed, using (2) we can write:

$$
\begin{aligned}
{\left.\left[\left(\vec{N}_{\tau}, L_{\tau}\right),\left(\vec{N}_{\tau}^{\prime}, L_{\tau}^{\prime}\right)\right]\right|_{\Sigma_{\tau}} } \\
=\left.\left[\vec{N}_{\tau}+L_{\tau} \hat{n}_{\tau}, \vec{N}_{\tau}^{\prime}+L_{\tau}^{\prime} \hat{n}_{\tau}\right]\right|_{\Sigma_{\tau}} \\
=\left[\vec{N}_{\tau}, \vec{N}_{\tau}^{\prime}\right]+\left.\left[\vec{N}_{\tau}, L_{\tau}^{\prime} \hat{n}_{\tau}\right]\right|_{\Sigma_{\tau}}+\left.\left[L_{\tau} \hat{n}_{\tau}, \vec{N}_{\tau}^{\prime}\right]\right|_{\Sigma_{\tau}}+\left.\left[L_{\tau} \hat{n}_{\tau}, L_{\tau}^{\prime} \hat{n}_{\tau}\right]\right|_{\Sigma_{\tau}} \\
=\left[\vec{N}_{\tau}, \vec{N}_{\tau}^{\prime}\right]+\vec{N}_{\tau}\left(L_{\tau}^{\prime}\right) \hat{n}_{\tau}+\left.L_{\tau}^{\prime}\left[\vec{N}_{\tau}, \hat{n}_{\tau}\right]\right|_{\Sigma_{\tau}}+\left.L_{\tau}\left[\hat{n}_{\tau}, \vec{N}_{\tau}^{\prime}\right]\right|_{\Sigma_{\tau}}-\vec{N}_{\tau}^{\prime}\left(L_{\tau}\right) \hat{n}_{\tau} \\
\quad+L_{\tau} \hat{n}_{\tau}\left(L_{\tau}^{\prime}\right) \hat{n}_{\tau}-L_{\tau}^{\prime} \hat{n}_{\tau}\left(L_{\tau}\right) \hat{n}_{\tau} .
\end{aligned}
$$

Now, since both evolution vector fields are $\Sigma_{\tau}$-Gaussian for any $\tau$, using (15) gives:

$$
\begin{aligned}
{\left.\left[\left(\vec{N}_{\tau}, L_{\tau}\right),\left(\vec{N}_{\tau}^{\prime}, L_{\tau}^{\prime}\right)\right]\right|_{\Sigma_{\tau}}=} & {\left[\vec{N}_{\tau}, \vec{N}_{\tau}^{\prime}\right]+L_{\tau} \operatorname{grad}_{\gamma_{\tau}} L_{\tau}^{\prime} } \\
& -L_{\tau}^{\prime} \operatorname{grad}_{\gamma_{\tau}} L_{\tau}+\left(\vec{N}_{\tau}\left(L_{\tau}^{\prime}\right)-\vec{N}_{\tau}^{\prime}\left(L_{\tau}\right)\right) \hat{n}_{\tau},
\end{aligned}
$$

which yields exactly the Poisson bracket structure of the ADM constraints (6)-(8). The Poisson bracket of the constraints is then naturally dependent on the slice on 
which it is calculated, in particular on its inherited Riemannian geometry. We can conclude that indeed the way in which the Poisson bracket of the geometrodynamical constraints close could not be any different, as we have just derived it from the general form of the theory, using only the infinitesimal version of our Postulate (Theorem 1) in the form of Eq. (15). In other words, if we only insist on the geometrodynamical framework as described in the introduction to be self consistent in the sense of our Postulate (Theorem 1), the Poisson bracket structure of the constraints depends on the slice in a fixed manner as expected, regardless of the specifics of the matter fields that one wishes to consider.

\section{Discussion}

The conclusion of this short paper is simply that if the generally relativistic generalization of the Hamiltonian field theory in the shape of geometrodynamics is to be consistent-in the sense of satisfying our Postulate 1-the Poisson bracket structure of the constraints that govern evolution cannot be any different. This perspective clarifies the two intriguing observations mentioned in the introduction, namely that:

(i) The bracket structure is insensitive to the matter content (as long as the coupling is non-derivative) - this is simply because so is our derivation of it.

(ii) Its origin is indeed purely geometric, with the link to dynamics given by our interpretation of the $\Sigma$-Gaussian vector fields (that was the missing element of [3]).

Our result is particularly interesting in the context of the third point mentioned in the introduction, namely the work of Hojman et al. [1], who reach the following conclusion:

Einsteinian geometrodynamics is the only (time-reversible) canonical representation of the set of generators of deformations of a space-like hypersurface embedded in a Riemannian spacetime, if the intrinsic metric of that hypersurface and a conjugate momentum are the sole canonical variables.

By 'Einsteinian geometrodynamics' they mean precisely the framework we described in Sect. 3 with the ADM constraints and, as is clear from the remaining of the sentence, they refer to the situation with no matter fields. 'Deformations of a space-like hypersurface embedded in a Riemannian spacetime' is the geometrical framework developed by Teitelboim in [2] and further clarified in [3] that allows to generate the bracket structure identical with the Poisson bracket structure of the ADM constraints as the commutator of the generators of such deformations. Confusingly, 'Riemannian spacetime' means what we now call a connected Lorentzian manifold. The term 'canonical representation' refers to the fact that the Poisson bracket structure of the constraints is assumed to be of this specific form. The cited claim can then be rephrased as: 
From the correct Poisson bracket structure of the pure geometrodynamical constraints and time-reversibility of the theory ADM gravity can be deduced.

The authors thus derive the gravitational constraints (4) and (5) from the following assumptions: (1) the general form of the theory as presented in our introduction, (2) time-reversibility and (3) the particular Poisson bracket structure of the constraints. We believe that we have improved this already remarkable result by recognizing the representation assumption (4) to be merely a consistency requirement, derivable from the correct interpretation of the geometrodynamical framework. In the light of our result the cited claim then yields:

Theorem 3 The only consistent time-reversal pure geometrodynamics is the ADM gravity.

The point we are making here is a bit subtle. The 'representation requirement' of [1] is motivated by (1) the fact that it holds for ADM gravity and (2) the geometric construction developed by Teitelboim [2]. It is already plausible to assume that the commutator structure of the generators of hyper-surface deformations of [2] is matched by the geometrodynamical evolution given by the Poisson brackets of the constraints. Our point is to further justify this insight by showing that it follows directly from the Postulate 1 , which we think should be seen as a part of the geometrodynamical framework. Indeed, as we argue in the Sect. 2, without it the interpretation of dynamical data would be ambiguous.

Acknowledgements This work was made possible thanks to the Heinrich Hertz Fellowship that I was awarded, which allowed me to become a part of the Lichtenberg Group for History and Philosophy of Physics, led by Prof. Dennis Lehmkuhl at the University of Bonn. I would also like to express my gratefulness to my $\mathrm{PhD}$ supervisor Prof. Marek Kuś for the freedom and encouragement he provided me with, as well as to Prof. Jerzy Kijowski for some very informative discussions. The presented ideas are inspired by the work of Prof. Alan Weinstein and Dr. Christian Blohman. I would also like to mention here my former supervisor, Prof. Klaas Landsman, thanks to whom my interest in this topic, and many others, have developed. My research was partly funded by NCN through the OPUS Grant No. 2017/27/B/ST2/02959.

Open Access This article is licensed under a Creative Commons Attribution 4.0 International License, which permits use, sharing, adaptation, distribution and reproduction in any medium or format, as long as you give appropriate credit to the original author(s) and the source, provide a link to the Creative Commons licence, and indicate if changes were made. The images or other third party material in this article are included in the article's Creative Commons licence, unless indicated otherwise in a credit line to the material. If material is not included in the article's Creative Commons licence and your intended use is not permitted by statutory regulation or exceeds the permitted use, you will need to obtain permission directly from the copyright holder. To view a copy of this licence, visit http://creativecommons.org/licen ses/by/4.0/.

\section{References}

1. Hojman, S.A., Kuchař, K., Teitelboim, C.: Geometrodynamics regained. Ann. Phys. 96, 88-135 (1976)

2. Teitelboim, C.: How commutators of constraints reflect the spacetime structure. Ann. Phys. 79, 542-557 (1973)

3. Blohmann, C., Fernandes, M., Weinstein, A.: Groupoid symmetry and constraints in general relativity. Commun. Contemp. Math. 15, 1250061 (2010) 
4. Głowacki, J.: Groupoid symmetry and constraints of General Relativity revisited, MSc thesis. https://www. researchgate.net/publication/332960753_Groupoid_symmetry_and_constraints_of_General_Relat ivity_revisited

5. Blohmann, C., Weinstein, A.: Hamiltonian Lie algebroids. https://ui.adsabs.harvard.edu/abs/2018arXiv1 81111109B

6. Wald, R.: General Relativity. Chicago University Press, Chicago (1984)

Publisher's Note Springer Nature remains neutral with regard to jurisdictional claims in published maps and institutional affiliations. 